\title{
Fingolimod (FTY720) Enhances Remyelination Following Demyelination of Organotypic Cerebellar
} Slices

\author{
Veronique E. Miron, ${ }^{* \dagger}$ Samuel K. Ludwin, ${ }^{\ddagger}$ \\ Peter J. Darlington, ${ }^{*}$ Andrew A. Jarjour, ${ }^{\dagger \S}$ \\ Betty Soliven, ${ }^{\pi}$ Timothy E. Kennedy, ${ }^{\dagger}$ \\ and Jack P. Antel ${ }^{\star}$

\begin{abstract}
From the Neuroimmunology Unit," and the Center for Neuronal Survival, ${ }^{\dagger}$ Mc Gill University, Montreal Neurological Institute, Montreal, Canada; the Department of Neuropathology, ${ }^{\ddagger}$ Queen's University, Kingston, Ontario, Canada; the Medical Research Council Centre for Regenerative Medicine, ${ }^{\S}$ The Queen's Medical Research Institute, University of Edinburgh, Edinburgh, United Kingdom; and the Department of Neurology, "Iniversity of Chicago, Chicago, Illinois
\end{abstract}

Remyelination, which occurs subsequent to demyelination, contributes to functional recovery and is mediated by oligodendrocyte progenitor cells (OPCs) that have differentiated into myelinating cells. Therapeutics that impact remyelination in the CNS could be critical determinants of long-term functional outcome in multiple sclerosis (MS). Fingolimod is a S1P receptor modulator in MS clinical trials due to systemic anti-inflammatory properties, yet may impact cells within the CNS by crossing the blood-brain barrier. Previous studies using isolated dissociated cultures indicate that neural cells express S1P receptors and respond to receptor engagement. Our objective was to assess the effects of fingolimod on myelinrelated processes within a multicellular environment that maintains physiological cell-cell interactions, using organotypic cerebellar slice cultures. Fingolimod treatment had no impact on myelin under basal conditions. Fingolimod treatment subsequent to lysolecithin-induced demyelination enhanced remyelination and process extension by OPCs and mature oligodendrocytes, while increasing microglia numbers and immunoreactivity for the astrocytic marker glial fibrillary acidic protein. The number of phagocytosing microglia was not increased by fingolimod. Using S1P receptor specific agonists and antagonists, we determined that fingolimod-induced effects on remyelination and astrogliosis were mediated primarily through S1P3 and
S1P5, whereas enhanced microgliosis was mediated through S1P1 and S1P5. Taken together, these data demonstrate that fingolimod modulates multiple neuroglial cell responses, resulting in enhanced remyelination in organotypic slice cultures that maintain the complex cellular interactions of the mammalian brain. (Am J Pathol 2010, 176:2682-2694; DOI: 10.2353/ajpath.2010.091234)

In the CNS, oligodendrocytes produce the myelin sheath, which forms an insulating layer around axons to enhance conduction velocity of electrical impulses. ${ }^{1-3}$ The maintenance of myelin requires continuous turnover of its components throughout life. ${ }^{4-5}$ Demyelinating insults contribute to axonal degeneration, functional impairments, and clinical disability in the autoimmune disease multiple sclerosis (MS). ${ }^{6-7}$ Neuropathological and magnetic resonancebased observations indicate that re-ensheathment of demyelinated axons, termed remyelination, occurs in demyelinated lesions in MS and may contribute to clinical remittance $^{8-9}$ Remyelination is critical to restore electrical impulse conduction and protect axons from degeneration or further injury. ${ }^{10-14}$ Experimental animal models of CNS demyelination indicate that remyelination is mediated by oligodendrocytes generated from oligodendrocyte progenitor cells (OPCs). ${ }^{15}$ Demyelinating models show that both astrocytes and microglia are critical for remyelination, with microglia contributing to clearance of debris and both cell types participating in creating an environment permissive for remyelination. ${ }^{16-19}$

Supported by Novartis Pharma (Basel, Switzlerland), the Multiple Sclerosis Society of Canada (J.P.A., T.E.K.), National Multiple Sclerosis Society (J.P.A.), and Canadian Institutes of Health Research (studentship to V.E.M.).

Accepted for publication February 16, 2010.

This research was presented at the 2009 American Academy of Neurology Annual Meeting with support from Novartis Pharma.

Supplemental material for this article can be found on http://ajp. amjpathol.org.

Address reprint requests to Dr. Jack P. Antel, Room 111, Neuroimmunology Unit, Montreal Neurological Institute, 3801 University St., Montreal, QC, Canada, H3A 2B4, E-mail: jack.antel@mcgill.ca. 
The eventual failure of remyelination that occurs as MS progresses may result from the generation of a nonpermissive environment or intrinsic deficits in the remyelinating cells. Therapeutic agents that impact on myelin maintenance and remyelination are predicted to be important determinants of long-term functional outcome in MS. Fingolimod (FTY720) is currently in clinical trials due to its anti-inflammatory properties, ${ }^{20}$ yet readily crosses the blood brain barrier. ${ }^{21}$ Analysis of the distribution of fingolimod orally administered to mice for 1 week indicated that the drug is sequestered in the brain parenchyma relative to the blood. ${ }^{22}$ Once fingolimod has entered the brain, it is phosphorylated to its biologically active form which then binds sphingosine-1-phosphate (S1P) receptors. ${ }^{23-24}$

Previous experiments have demonstrated that isolated neuroglial cell types in dissociated cultures can respond to S1P receptor engagement. Both human and rodent cells of the oligodendroglial lineage are able to respond to fingolimod due to the expression of S1P receptor isoforms 1,3 , and $5 .{ }^{25-28} \mathrm{~S} 1 \mathrm{P}$ or fingolimod treatment of rodent pre-OLGs (O4+/Galactocerebroside-) or human fetal OPCs (A2B5+), respectively, leads to transient retraction of processes via S1P5. ${ }^{27,29}$ High doses of fingolimod synergistically enhance process branching and elongation by rat OPCs when co-applied with neurotrophic factors. ${ }^{30}$ Fingolimod can either promote or inhibit rodent and human OPC differentiation in a dose-dependent manner. ${ }^{27,30}$ Whereas the cytoskeletal dynamics of rat mature OLGs in dispersed cell culture are not influenced by fingolimod, this agent induces a cyclical regulation of S1P1-dependent membrane elaboration and S1P3/5-dependent membrane retraction in human mature OLGs over time. ${ }^{28}$ Astrocytes and microglia also respond to S1P-directed signaling. S1P receptor engagement in astrocytes leads to extracellular signal-regulated kinase-1/2 activation, resulting in proliferation and neurotrophic factor production. ${ }^{31-33}$ Astrocytes additionally produce platelet-derived growth factor (PDGF), which can alter S1P receptor levels. ${ }^{34}$ Fingolimod can inhibit macrophage infiltration following traumatic brain injury. ${ }^{35}$

Although the effects of S1P receptor signaling on individual neural cell types has been described, the direct influence of fingolimod on neuroglial cells and myelinrelated processes in a physiological environment containing all of the resident CNS elements, and the contribution of individual S1P receptors to these responses, have yet to be investigated. Here, we delineate the consequence of fingolimod treatment on myelin maintenance and remyelination, using an in vitro model that recapitulates myelin-axon and cell-cell interactions. Rodents orally administered $0.3 \mathrm{mg} / \mathrm{kg}$ of fingolimod daily over the long-term were shown to have micromolar concentrations of the active form of the drug within the brain parenchyma, whereas subnanomolar concentrations were measured in the cerebrospinal fluid. ${ }^{22}$ Newborn mouse organotypic cerebellar slice cultures were treated with the active phosphorylated form of fingolimod at physiological doses representative of those measured in the brain $(1 \mu \mathrm{mol} / \mathrm{L})$ and cerebrospinal fluid (100 pmol/L). We have previously demonstrated that human neural cell properties are modulated in vitro by fingolimod doses within the range of $100 \mathrm{pmol} / \mathrm{L}$ to $1 \mu \mathrm{mol} / \mathrm{L} .{ }^{27,28} \mathrm{We}$ had observed that cells responded similarly when treated with $100 \mathrm{pmol} / \mathrm{L}$ to $1 \mathrm{nmol} / \mathrm{L}$ of fingolimod, or with $10 \mathrm{nmol} / \mathrm{L}$ to $1 \mu \mathrm{mol} / \mathrm{L}$ of the drug. ${ }^{28} \mathrm{We}$ report that fingolimod influences cells of the oligodendroglial lineage, microglia, and astrocytes, resulting in enhanced remyelination in the absence of a systemic immune system.

\section{Materials and Methods}

\section{Animals and Tissue Preparation}

Newborn (P0) wild-type CD1 mouse pups were obtained from Charles River (Quebec, Canada). All procedures were performed in accordance with the Canadian Council on Animal Care guidelines. Slices were obtained and cultured as previously described. ${ }^{36}$ Cerebellum and attached hindbrain were extracted in L15 media and cut into $300 \mu \mathrm{m}$ sagittal sections using a Mcllwain tissue chopper. Slices were separated and plated on a Millipore-Millicel-CM culture insert (Fisher Scientific, Ottawa, ON, Canada) in 6-well cell culture plates, with 6 slices per insert. Culture media was composed of $50 \%$ minimal essential media, 25\% heat-inactivated horse serum, 25\% Earle's balanced salt solution, $6.5 \mathrm{mg} / \mathrm{ml}$ glucose (SigmaAldrich, St-Louis, MO), and penicillin and glutamine supplements (All from Invitrogen, Carlsbad, CA). Membranes were transferred into fresh media every 2 to 3 days. Slices were left untreated for 21 days in vitro (DIV) to allow clearance of debris and myelination to occur. ${ }^{37}$

\section{Pharmacological Treatment}

The active phosphorylated form of fingolimod was provided by Novartis Pharma (Basel, Switzerland) and was reconstituted in dimethylsulfoxide/50 $\mathrm{mmol} / \mathrm{L} \mathrm{HCl}$. Fingolimod was diluted in basal culture media and replaced every 2 to 3 days. For myelin maintenance studies, cultures were treated for three subsequent weeks with fingolimod. For remyelination studies, cultures were demyelinated with lysolecithin overnight $(0.5 \mathrm{mg} / \mathrm{ml})$, washed with PBS twice for 10 minutes, allowed to recover in basal culture media for 2 days, then treated with fingolimod for the next 2 weeks (14 DIV). To investigate the response to fingolimod independent from demyelination, control cultures were allowed to recover for 14 DIV postlysolecithin, then either left untreated for another 14 DIV (control 28 DIV postlysolecithin), or treated with fingolimod for 14 DIV (total 28 DIV postlysolecithin). Cultures treated with the vehicle used to reconstitute fingolimod showed no significant effects on myelin. To determine the mechanism of fingolimod-induced effects, demyelinated cultures were co-treated with fingolimod (100 pmol/L) and a S1P1 antagonist W123 (1 $\mu \mathrm{mol} / \mathrm{L}$; Cayman Chemicals, Ann Arbor, $\mathrm{MI})$ or a S1P3/5 pathway antagonist suramin $(100 \mathrm{nmol} / \mathrm{L}$; EMD Bioscience, San Diego, CA). ${ }^{27-28}$ More specific S1P receptor agonists were used to determine whether activation via specific S1P receptors could mimic the fingoli- 
mod-induced effects, including a S1P1 agonist SEW2871 $(100 \mathrm{nmol} / \mathrm{L} \text {; EMD Bioscience })^{27-28}$ and a S1P5 agonist (100 nmol/L; provided by Novartis Pharma). The experiments were performed on six different preparations.

\section{Whole Mount Immunohistochemistry}

Following two quick washes in PBS, slices were fixed in $1 \%$ paraformaldehyde (PFA) for 15 minutes for node of Ranvier staining (Caspr and sodium channel), or 4\% PFA for 1 hour for all other stains, then washed twice in PBS. Slices were cut from the membrane and blocked for 3 hours at room temperature in $1 \mathrm{mmol} / \mathrm{L}$ HEPES, $2 \%$ heatinactivated horse serum, 10\% heat-inactivated goat serum, $1 \%$ bovine serum albumin, and $0.25 \%$ Triton X-100 in Hank's Balanced Salt Solution. Primary antibodies diluted in block solution were applied for 48 hours at $4^{\circ} \mathrm{C}$. These include mouse anti-myelin basic protein (MBP, 1:500; Sternberger Monoclonals Inc., Lutherville, MD), rabbit anti-platelet-derived growth factor receptor $\alpha$ (PDGF $\alpha$ R, 1:50; Santa-Cruz Biotechnology, Santa Cruz, $\mathrm{CA})$, mouse anti-neurite outgrowth inhibitor protein $A$ (NogoA, 1:1000; provided by Dr.Martin Schwab, University of Zurich), polyclonal chicken anti-neurofilament medium chain (NFM, 1:2000; EnCor Biotech, Alachua, FL), mouse anti- $\beta$ tubulin III ( $\beta$-TublII, 1:400; Sigma), rabbit anti-ionized calcium binding adaptor molecule 1 (IBA-1, 1:500; Wako Chemicals USA, Richmond, VA), rabbit antiglial fibrillary acid protein (GFAP, 1:200; NeoMarkers, Fremont, CA), mouse monoclonal pan anti-sodium channel (1:1000; Sigma), and rabbit anti-contactin associated protein (Caspr, 1:10 000; provided by Dr. David Colman, McGill University). Slices were washed thrice in PBS with $0.05 \%$ Triton $X-100$ for 1 hour on a rotary shaker then incubated in secondary antibody diluted in block overnight at $4^{\circ} \mathrm{C}$. Secondary antibodies include goat antimouse Cy3 (1:500; Jackson Immunoresearch, West Grove, PA), donkey anti-chicken-Alexa-488 (1:70; Molecular Probes, Eugene, OR), goat anti-mouse Alexa-488 (1:500; Molecular Probes), and goat anti-rabbit Cy3 (1: 250; Jackson ImmunoResearch). Slices were counterstained with nuclear dye Topro-3 (1:1000; Molecular Probes), washed thrice, and mounted on glass slides (Fisher Scientific) using Fluoromount G (Southern Biotech, Birmingham, AL).

\section{Immunohistochemical Analysis}

Single optical $\times 63$ or $\times 100$ objective images were acquired using a Zeiss LSM 510 microscope (Peabody, MA). Two to three representative images were acquired per stain per slice. The range of the slice was determined using Z-stack imaging at 1- to 3- $\mu \mathrm{m}$ intervals. By Z-stack analysis, we found that slices thinned to approximately 30 $\mu \mathrm{m}$ in culture. Analysis demonstrated that a fully myelinated unmanipulated slice had three distinct regions in the $Z$ plane that differed in their cellular composition and appearance: the first $\sim 1$ to $5 \mu \mathrm{m}$ ('top') is where the majority of the myelin, axons, OLGs, OPCs, and astrocytes reside; the next $\sim 6$ to $23 \mu \mathrm{m}$ ('middle') have the same composition as the 'top' layer however at lesser amounts; the last $\sim 24$ to $30 \mu \mathrm{m}$ ('bottom') was mostly devoid of all cellular components except microglia, which were also found throughout other depths of the slice albeit at lower levels. Quantification was performed separately in the top and middle of the slice where the myelin was located, to control for sampling error and variation in thickness of each slice.

The area of MBP or GFAP immunostaining $\left(\mu \mathrm{m}^{2}\right)$ per $\times 63$ objective image was determined using Scion Image software (Scion Corporation, Frederick, MD). The numbers of PDGF $\alpha$ R, NogoA, and IBA-1 immunopositive cells were counted in a blinded fashion either from representative $\times 63$ objective images or a series of images derived from Z-stack imaging. The number of nodes of Ranvier per $\times 100$ objective image was determined by counting areas of condensed sodium channel immunostaining flanked by Caspr immunostaining. The diameter of the Node of Ranvier $(\mu \mathrm{m})$ was evaluated by calibrating $\times 100$ objective images and measuring the length of condensed sodium channel immunostaining using ImageJ software (U. S. National Institutes of Health, Bethesda, Maryland, http://rsb.info.nih.gov/ij).

\section{Phagocytosis Assay}

Phagocytosis of E. Coli-coated beads was used to measure phagocytic potential of microglia in the slices. A $2 \mu$ l drop of rhodamine-conjugated E. Coli bioparticles (1 mg/ $\mathrm{ml}$; Invitrogen) reconstituted in culture media was added directly on top of the slice. Slices were incubated for 3 hours at $37^{\circ} \mathrm{C}$, then washed in PBS twice for 10 minutes, and fixed in 4\% PFA for 1 hour. Slices were then stained against a microglial marker, IBA-1. Slices were kept in the dark as much as possible during the fixation and immunohistochemical staining processes. Absolute numbers of phagocytic microglia were taken to be IBA-1 immunopositive cells with red signal within the cell body, and were counted in a blinded manner in images obtained from Z-stacks. Phagocytosis assays were performed using one slice culture preparation.

\section{Bromodeoxyuridine Incorporation}

Bromodeoxyuridine (BrdU) incorporation and immunohistochemistry was performed as previously described. ${ }^{38}$ Briefly, BrdU was diluted in culture media to a concentration of $10 \mu \mathrm{mol} / \mathrm{L}$ and added to slices for 24 hours before fixation in 4\% PFA for 1 hour. Slices were denatured with $2 \mathrm{~N} \mathrm{HCl}$ for 45 minutes, rinsed in PBS for 1 minute, then incubated with mouse anti-BrdU for 48 hours (1:40; Sigma) and goat anti-mouse secondary antibody overnight at $4^{\circ} \mathrm{C}$.

\section{Statistical Analysis}

Values are represented as means \pm SEM, and some are normalized to control cultures at the respective time point. Statistical analyses were performed using one-way analysis of variance (Newman-Keuls and Dunns multiple 
comparison tests), unpaired two-tailed Student's $t$-test, and Mann-Whitney test. $P$ values less than 0.05 were taken to represent significant differences between treatment groups.

\section{Electron Microscopy}

Electron microscopy was used to confirm the integrity of myelin and to assess actual remyelination as documented by thin myelin sheaths. Cerebellar slices were fixed overnight with $2.5 \%$ glutaraldehye in $0.1 \mathrm{~mol} / \mathrm{L}$ sodium cacodylate buffer then cut out of the surrounding membrane, subsequently osmicated with potassium ferrocyanide-reduced $1 \%$ osmium tetroxide solution for 1 hour, and dehydrated with successive 10-minute washes in increasing concentrations of ethanol. Slices were infiltrated for 1 hour with 1:1, 1:2, and 1:3 ethanol: Epon blends, followed by infiltration with pure Epon. Slices were embedded in Epon in a plastic BEEM capsule. Sections of 70 to $100 \mu \mathrm{m}$ were cut onto 200 mesh copper grids and stained with $4 \%$ uranyl acetate and Reynold's lead citrate. Semithin sections of $1 \mu \mathrm{m}$ were stained with toluidine-blue for neuropathological analysis.

\section{Results}

\section{Characterization of Organotypic Cerebellar Slice Cultures}

To assess the role of fingolimod on remyelination, we elected to use organotypic cerebellar slice cultures due to previous studies demonstrating robust and reproducible myelination along definable axons in slices derived from this brain region, and enhanced plasticity and resistance to mechanical trauma at this age. ${ }^{36,37,39}$ Organotypic slices retain the astrocyte and microglia elements that allow us to identify their contribution to myelinationassociated processes, but avoid the complex systemic immune system interactions observed in intact animals. Confocal analysis of untreated slice cultures at the temporal onset of pharmacological manipulation (21 DIV) indicated that cultures were comprised of myelin $(\mathrm{MBP}+)$, mature oligodendrocytes (NogoA + ), neurons (NFM+, $\beta$ TubllI+), OPCs (PDGF $\alpha \mathrm{R}+$ ), astrocytes (GFAP + ), and microglia (IBA-1+) (see Supplemental Figure 1A at http://ajp. amjpathol.org). There was a significant co-localization between myelin (MBP) and axons (NFM) in the X, Y, and Z axes (see Supplemental Figure S1, A and B, at http:// ajp.amjpathol.org). Cross-sectional analysis confirmed that MBP+ fibers ensheathed NFM+ axons (see Supplemental Figure S1B at http://ajp.amjpathol.org). Not all NFM immunopositive fibers were associated with MBP staining, consistent with not all axons being myelinated in organotypic cerebellar slices. ${ }^{37}$ Ultrastructural analysis demonstrated that the myelin was compact and organized appropriately into nodes of Ranvier (see Supplemental Figure S1C at http://ajp.amjpathol.org), and was therefore mature and of good integrity.

\section{Effects of Fingolimod on Myelin Maintenance and Oligodendroglia}

Following recovery from dissection, cultures were treated with the active phosphorylated form of fingolimod for the subsequent 21 DIV then analyzed immunohistochemically. Fingolimod treatment ( $100 \mathrm{pmol} / \mathrm{L}, 1 \mu \mathrm{mol} / \mathrm{L}$ ) did not significantly affect the area of MBP staining per field relative to untreated cultures $(0.97 \pm 0.3,0.95 \pm$ 0.2 fold over control, respectively), consistent with no deleterious effects of fingolimod on myelin maintenance (Figure 1A). We next investigated the effect of fingolimod on mature OLGs, labeled with the OLG cell body marker NogoA, ${ }^{40-41}$ and on OPCs identified by PDGF $\alpha$ R expression. Fingolimod treatment $(100 \mathrm{pmol} / \mathrm{L}$, $1 \mu \mathrm{mol} / \mathrm{L}$ ) induced membrane elaboration and process outgrowth by OLGs (Figure 1B) and OPCs (Figure 1C), respectively, relative to control. Fingolimod (100 pmol/L, $1 \mu \mathrm{mol} / \mathrm{L}$ ) had no significant impact on the absolute number of PDGF $\alpha$ R + cells, although a trend toward an increase was observed with the lowest dose $(12 \pm 2$ and $6 \pm 1$ per field, respectively, relative to $8 \pm 0$ for control) (Figure 1D). Fingolimod treatment for 21 DIV (100 pmol/L) caused a significant decrease in numbers of microglia (IBA-1+) from $7 \pm 1$ to $1 \pm 0$ per field, and a significant decrease in the area of GFAP immunostaining (from $3886 \pm 579 \mu \mathrm{m}^{2}$ to $2192 \pm 492$ ).

\section{Effects of Fingolimod on Remyelination and Oligodendroglial Lineage Cells Following Demyelination: Characterization of Lysolecithin-Induced Demyelination}

The bioactive lipid lysolecithin (Iysophosphatidylcholine) induces demyelination when focally injected into white matter tracts such as the cerebellar peduncle and spinal cord, ${ }^{42-43}$ or applied to organotypic slice cultures. ${ }^{37}$ Following 21 DIV of postisolation recovery, slices were treated with $0.5 \mathrm{mg} / \mathrm{ml}$ lysolecithin for 16 hours, which induces demyelination of organotypic slices by 2 days postapplication while minimizing concomitant axonal injury. ${ }^{37}$ Analysis of cultures 2 days after application of lysolecithin indicated a notable decrease in the area of MBP staining $(0.23 \pm 0.18$-fold over normally myelinated control at 21 DIV) such that immunopositive staining was associated with myelin debris and ensheathed axons were rarely observed (Figure 2, A and B). In comparison with toluidine-blue stained semithin sections from fully myelinated controls (Figure $2 \mathrm{C}$ ), sections from lysolecithin-treated cultures demonstrated many demyelinated fibers, few residually myelinated fibers, and accumulation of myelin debris (Figure 2D). NFM staining was not adversely affected by lysolecithin treatment indicating a sparing of axons (Figure 2A). Fully myelinated cultures at 21 DIV demonstrated appropriately formed nodes of Ranvier characterized by paranodal Caspr immunostaining flanking sodium channels localized to the node itself (Figure 2E). Demyelination with lysolecithin caused a lengthening of the distribution of sodium channels mark- 
A

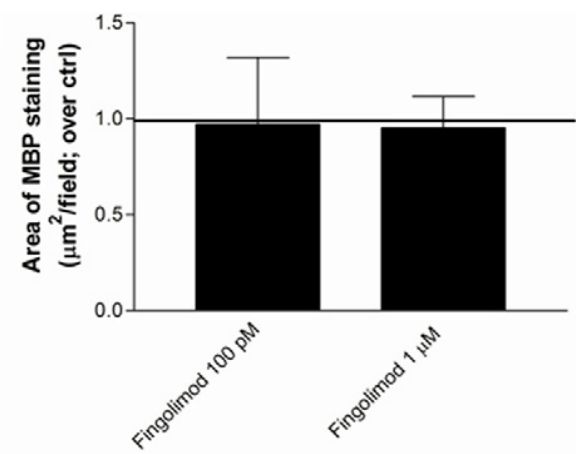

B

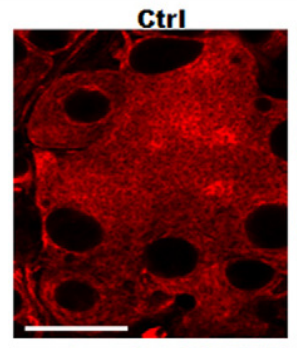

Fingolimod 100pM

C

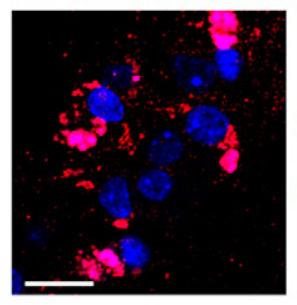

D

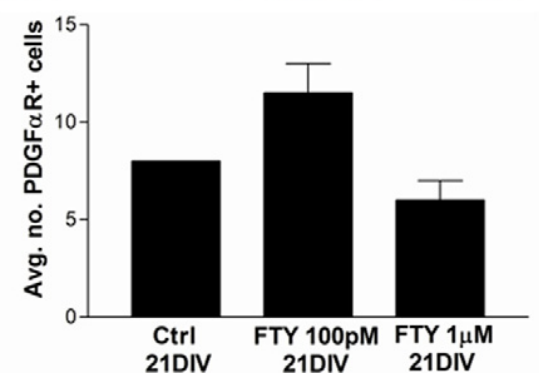

Figure 1. Effect of fingolimod on myelin and oligodendroglial lineage cells under nonpathological conditions. A: Quantification of amount of myelin expressed as area of MBP immunostaining $\left(\mu \mathrm{m}^{2}\right)$ per $\times 63$ objective image normalized to untreated control culture values at 35 days in vitro (DIV). Treatment with fingolimod $(100 \mathrm{pmol} / \mathrm{L}, 1 \mu \mathrm{mol} / \mathrm{L})$ for $21 \mathrm{DIV}$ did not significantly affect the amount of myelin in the cultures. B: Representative confocal images of organotypic cerebellar slice cultures at 35 DIV immunostained against the oligodendrocyte marker, neurite outgrowth inhibitor protein A (NogoA; red). Untreated cultures were characterized by NogoA positive mature oligodendrocytes that demonstrated some membrane fusion. Treatment with fingolimod (100 pmol/L) for 21 DIV induced process outgrowth and membrane elaboration of NogoA positive cells relative to control. Scale bar $=20 \mu \mathrm{m}$. C: Representative confocal images of organotypic cerebellar slices at 35 DIV immunostained against the oligodendrocyte progenitor cell (OPC) marker platelet-derived growth factor receptor $\alpha$ (PDGF $\alpha \mathrm{R}$; red) and counterstained with the nuclear Topro-3 (blue). Some immunopositive cells were observed in untreated cultures. Treatment with fingolimod (100 $\mathrm{pmol} / \mathrm{L}$ ) for 21 DIV caused process outgrowth in OPCs. Scale bar $=20 \mu \mathrm{m}$. D: Quantification of absolute numbers of PDGF $\alpha$ R + OPCs per $\times 63$ objective image represented as average \pm SEM, at 35 DIV. There was a trend increase in OPC numbers with $100 \mathrm{pmol} / \mathrm{L}$ fingolimod treatment for $21 \mathrm{DIV}$.

ing the node (from $0.31 \pm 0.06$ to $11.24 \pm 1.98 \mu \mathrm{m}^{2}$; Figure 2F), a decrease in the average number of nodes of Ranvier (from $22 \pm 3$ to $7 \pm 2$ nodes per field; Figure $2 \mathrm{G}$ ), and a dispersion of Caspr immunostaining; the latter has been described in demyelinated MS lesions. ${ }^{44}$

\section{Effects of Fingolimod on Recovery of Myelin Following Lysolecithin-Induced Demyelination}

Analysis of control cultures at 14 DIV postlysolecithin (total 35 DIV) indicated that some recovery of MBP staining and ensheathment had occurred (1.4 \pm 0.42 compared with 2 days postlysolecithin; Figure 3A); however, remyelination was incomplete at this time point, as has been previously documented in this culture system. ${ }^{37}$ Application of fingolimod (100 pmol/L, $1 \mu \mathrm{mol} / \mathrm{L}$ ) for 14 DIV following lysolecithin-induced demyelination caused a significant increase in the area of MBP staining per field relative to remyelinating control, indicative of enhanced remyelination $(1.97 \pm 0.33$ and $2.00 \pm 0.40$-fold over control at 35 DIV, respectively) (Figure $3, \mathrm{~A}$ and $\mathrm{B}$ ). Ultrastructural evaluation of fingolimod-treated cultures revealed many thin myelin sheaths, some of which were associated with paranodes suggestive of functional remyelination (Figure 3C). These cultures maintained appropriate cell-cell contacts and cyto-architecture as demonstrated by synaptic associations and adjacent localization of astrocytes (Figure 3D). Whereas control semithin toluidine-blue stained sections were characterized by many unmyelinated yet apparently healthy fibers (Figure 3E), sections from fingolimod-treated cultures revealed the presence of many thin myelin sheaths, some thicker myelin sheaths, and healthy neurons and oligodendrocytes (Figure 3F). As apparent in toluidine blue stained sections, both control and fingolimod-treated slices were characterized by the presence of phagocytosed myelin debris; we could not appreciate any qualitative difference in the amount of MBP-immunoreactive debris found in fingolimod-treated cultures compared with control cultures at 14 DIV postdemyelination.

A time course analysis of remyelination was determined with various fingolimod treatment durations by MBP immunohistochemistry. Remyelination was initiated as early as 5 DIV postdemyelination in both control and fingolimod-treated cultures. Although the area of MBP immunostaining was not significantly different between fingolimod treatment and control at both 5 and 12 DIV (data not shown), it was only significantly enhanced with fingolimod treatment at 14 DIV postlysolecithin (Figure 3 , $A$ and $B$ ). This finding indicates that the fingolimod-induced increase in remyelination occurred between 12 and 14 DIV following demyelination.

\section{Effects of Fingolimod on Oligodendroglia During Remyelination}

Fully myelinated cultures at 21 DIV were characterized by many NogoA + mature OLGs (Figure 4A). Lysolecithin-induced demyelination was associated with a loss of NogoA immunostaining. Cells positive for another mature OLGassociated cytoplasmic protein, adenomatous polyposis coli/CC1, could still be observed under demyelinating conditions (data not shown) suggesting that demyelination eliminated myelin-associated NogoA but did not kill all OLGs (Figure 4A). Control remyelinating cultures and fingolimod-treated cultures demonstrated a recovery of No- 

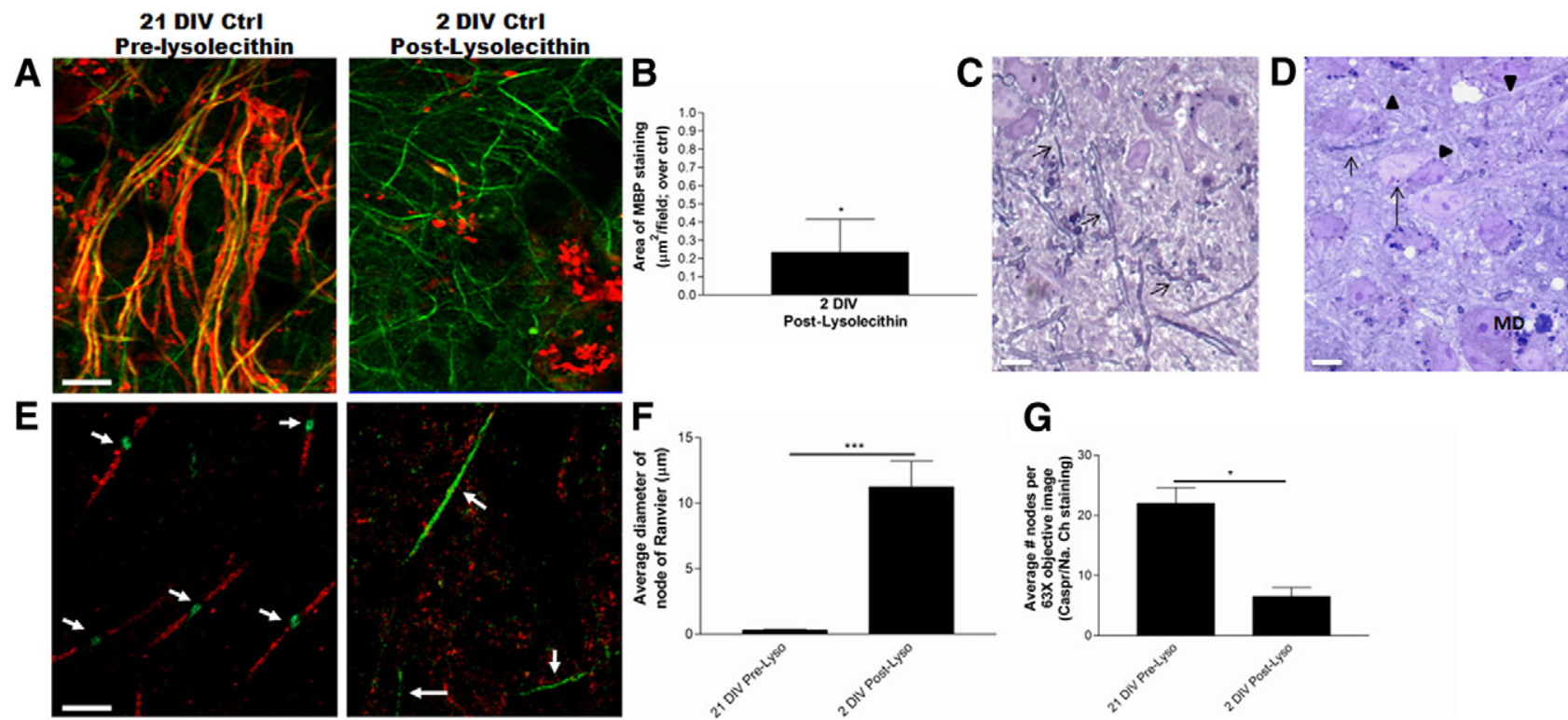

Figure 2. Characteristics of lysolecithin-induced demyelination of slices. A: Representative images of slices at 21 DIV (pre-demyelination) and 2 DIV postlysolecithin induced demyelination (23 DIV total), immunostained against myelin (MBP; red) and axons (NFM; green). Following lysolecithin treatment, MBP immunostaining is not associated with axons and likely reflects myelin debris. Scale bar $=20 \mu \mathrm{m}$. B: Quantification of amount of myelin expressed as area of MBP immunostaining $\left(\mu \mathrm{m}^{2}\right)$ per $\times 63$ objective image normalized to untreated control culture values at 21 DIV. Treatment with lysolecithin decreased the area of MBP staining. ${ }^{*} P<0.05$. C: Representative toluidine-blue stained section of culture at 21 DIV before demyelination. Arrows indicate myelinated fibers. Scale bar $=5 \mu \mathrm{m}$. D: Representative toluidine-blue stained section of lysolecithin-treated culture 2 DIV postdemyelination demonstrates some residually myelinated fibers (short arrow), many demyelinated axons (arrowheads), and some neurons showing some degenerative changes (long arrow). Myelin debris in the form of small blue stained collections and granules is seen both within and outside of cells (MD). Scale bar $=5 \mu \mathrm{m}$. E: Immunostaining of cultures pre-lysolecithin at 21 DIV demonstrates paranodal protein Caspr (red) flanking nodal aggregations of sodium channels (green), suggestive of proper node of Ranvier formation (arrows). Lysolecithin-induced demyelination induced a dispersion of Caspr immunostaining (red) and expansion of sodium channels along the axon (green; arrows). Scale bar $=10 \mu \mathrm{m}$. F: Average diameter of nodes of Ranvier $(\mu \mathrm{m})$ as measured by length of sodium channel aggregation \pm SEM, in cultures at 21 DIV (pre-lysolecithin) or 2 DIV postlysolecithin-induced demyelination. Demyelination induced a significant increase in the length of the node due to lateral migration of sodium channels. ${ }^{* * * *} P<0.001$. G: Average number of nodes of Ranvier per $\times 63$ objective image \pm SEM, in cultures at 21 DIV (pre-lysolecithin) or 2 DIV postlysolecithin-induced demyelination. Demyelination induced a significant decrease in the number of nodes of Ranvier. ${ }^{*} P<0.05$.

goA immunostaining over 14 DIV of postlysolecithin recovery (Figure 4A). At 14 DIV postlysolecithin, fingolimod treatment (100 $\mathrm{pmol} / \mathrm{L}, 1 \mu \mathrm{mol} / \mathrm{L})$ also induced a robust outgrowth and elaboration of NogoA + membranes compared with remyelinating control (Figure 4A).

Lysolecithin treatment caused a significant increase in absolute numbers of PDGF $\alpha$ R + OPCs $(17 \pm 2$ cells/ field), as compared with before lysolecithin treatment (control at 21 DIV; $3 \pm 0$ cells/field) (Figure 4, B and C). The absolute number of OPCs was significantly decreased in control cultures at 14 DIV postlysolecithin (5 \pm 0 cells/field; Figure 4C), suggestive of initial differentiation and loss of PDGF $\alpha$ R expression. The low dose of fingolimod (100 pmol/L) applied during 14 DIV postlysolecithin induced process extension in OPCs (Figure 4B) and caused a trend toward a relative increase in OPC numbers (9 \pm 1 cells/field; Figure 4C).

\section{Effects of Fingolimod on Microglia and Astrocytes During Remyelination}

To further evaluate the effects of fingolimod on neuroglial cells in the context of enhanced remyelination, effects on microglia and astrocytes were investigated. A time course analysis demonstrated that average numbers of IBA-1+ microglia peaked at 2 DIV postlysolecithin, from $1 \pm 0$ to $4 \pm 1 \mathrm{IBA}-1+$ cells/field (Figure 5, A and B). These numbers decreased by 5 DIV postlysolecithin and were maintained at this level by 9 DIV and 14 DIV postlysolecithin (Figure 5B), and may reflect the migration of microglia out of the slice. Accordingly, there was a slight increase in the number of IBA-1+ cells at the edge of the slice in these cultures (average 14 per field) compared with 2 DIV postlysolecithin (average 10 per field).

Fingolimod-treated cultures (100 pmol/L) had a significant increase in microglia at 5 DIV postlysolecithin (5 \pm 1 IBA-1+ cells/field compared with $2 \pm 1$ for control) (Figure 5B). This was further increased by 9 DIV postlysolecithin (14 \pm 2 compared with $2 \pm 1$ for control), and maintained by 14 DIV postlysolecithin ( $8 \pm 0$ relative to $3 \pm 0$ for control; Figure $5 \mathrm{~A}, \mathrm{~B})$. To determine whether this microglial response in the presence of fingolimod could be attributed to an enhanced response to demyelination, cultures were left untreated for 14 DIV postlysolecithin then subjected to another 14 DIV of fingolimod treatment (100 pmol/L; total 28 DIV postlysolecithin). These cultures still demonstrated a significant increase in microglia cell numbers $(13 \pm 1 \mathrm{IBA}-1+$ cells/field $)$ similar to that observed when fingolimod was applied for 14 DIV immediately postlysolecithin (10 \pm 1 ) (Figure 5B). Significantly more microglia were noted in this condition than in control cultures at 28 DIV postlysolecithin (6 \pm 1 ; Figure 5B), thereby dissociating this fingolimod-induced effect from a response to demyelination.

When assessing astrocytic reactivity, the area of GFAP immunostaining also peaked at 2 DIV postlysolecithin 
A

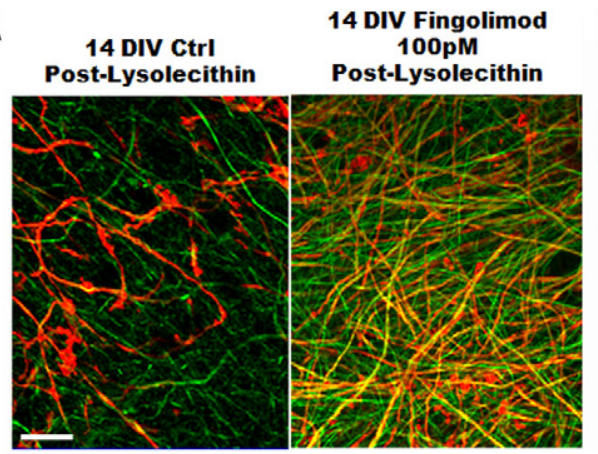

B
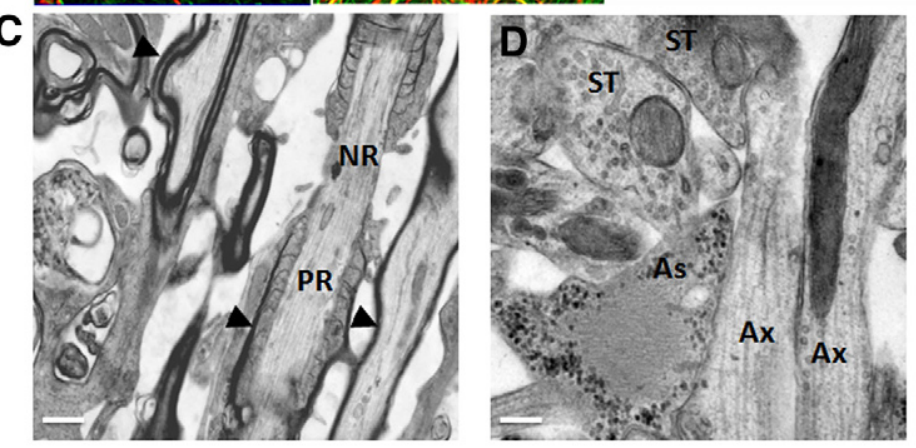

E

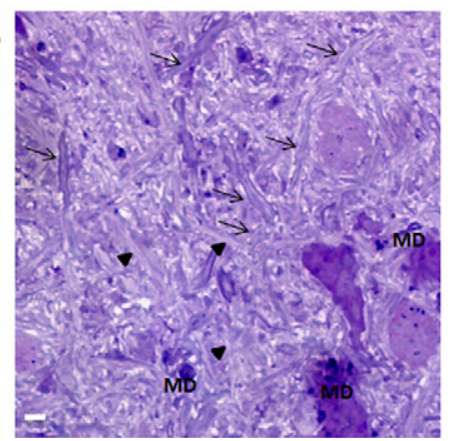

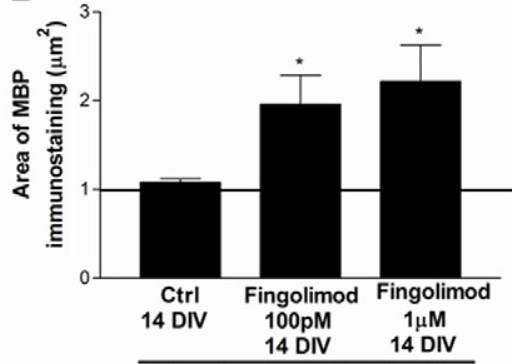

Treatment post-lysolecithin

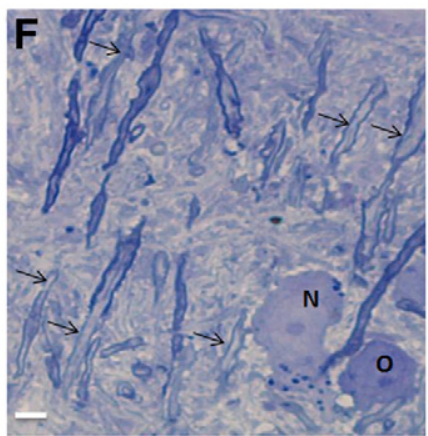

Figure 3. Effects of fingolimod on remyelination following lysolecithin-induced demyelination. A: Representative images of demyelinated slices, control or treated with fingolimod (100 $\mathrm{pmol} / \mathrm{L}$ ) for $14 \mathrm{DIV}$ postlysolecithin, immunostained against myelin (MBP; red) and axons (NFM; green). Fingolimod increases the amount of myelin associated with axons compared with control. Scale bar $=20 \mu \mathrm{mol} / \mathrm{L}$. B: Quantification of amount of myelin expressed as area of MBP immunostaining $\left(\mu \mathrm{m}^{2}\right)$ per $\times 63$ objective image normalized to control culture values at 14 DIV postlysolecithin. Fingolimod treatment (100 $\mathrm{pmol} / \mathrm{L}, 1 \mu \mathrm{mol} / \mathrm{L}$ ) for $14 \mathrm{DIV}$ postlysolecithin significantly increases the amount of myelin compared with control cultures. ${ }^{*} P<0.05$. C: Electron micrograph of demyelinated slice treated with $100 \mathrm{pmol} / \mathrm{L}$ fingolimod for $14 \mathrm{DIV}$ postdemyelination indicates thin myelin sheaths (arrows) suggestive of remyelination. Thin myelin sheaths at the paranode (PR) adjacent to the node of Ranvier (NR) are suggestive functional remyelination. Scale bar $=1 \mu \mathrm{m}$. D: Electron micrograph of demyelinated slice treated with $100 \mathrm{pmol} / \mathrm{L}$ fingolimod for 14 DIV postdemyelination indicates maintenance of appropriate cytoarchitecture, with synaptic terminals (ST) onto an axon (Ax) complete with synaptic vesicles, with an adjacent astrocyte (As) likely mediating homeostatic functions at the synapse. Scale bar $=0.5 \mu \mathrm{m}$. E: Toluidine-blue stained image of demyelinated slice allowed to recover for 14DIV postdemyelination. Arrowheads indicate demyelinated axons. Arrows indicate thin myelin sheaths suggestive of remyelination. Scale bar $=5 \mu \mathrm{m}$. MD, myelin debris F: Toluidine-blue stained image of demyelinated slice treated with $100 \mathrm{pmol} / \mathrm{L}$ fingolimod for $14 \mathrm{DIV}$ postdemyelination demonstrates a healthy neuron $(\mathrm{N})$ and oligodendrocyte $(\mathrm{O})$, as well as many thin myelin sheaths (arrows) indicating remyelination. Scale bar $=5 \mu \mathrm{m}$. $\left(2759 \pm 591 \mu \mathrm{m}^{2}\right)$ compared with before demyelination $\left(624 \pm 220 \mu \mathrm{m}^{2}\right)$ and subsided over the subsequent 14 DIV (1354 $\pm 550 \mu \mathrm{m}^{2}$; Figure 5, C and D). Similar to the effect on microglia cell numbers, fingolimod treatment (100 pmol/L) for 14 DIV postlysolecithin was associated with an increased area of GFAP immunostaining (4870 \pm $83 \mu \mathrm{m}^{2}$; Figure 5, C and D). Consistent with the finding that both astrocytes and microglia were increased in cultures treated with fingolimod for 14 DIV postlysolecithin, we observed a significant increase in cells that had incorporated BrdU relative to remyelinating control (from $0.3 \pm 0.2$ to $5 \pm 1 \mathrm{BrdU}+$ cells/field). The increased numbers of microglia and increased immunoreactivity for an astrocytic marker observed with fingolimod at 14 DIV postlysolecithin coincided with the time when a significant enhancement of remyelination was observed.

Previous studies have highlighted the importance of phagocytosis of myelin debris in allowing remyelination to proceed efficiently. ${ }^{17,39}$ Given the increased numbers of microglia observed in demyelinated slices treated with fingolimod for 14 DIV postlysolecithin, we determined whether fingolimod may have enhanced the phagocytic potential of microglia. Rhodamine-conjugated E. Colicoated beads were applied to the cultures for 3 hours and numbers of IBA-1 and E. Coli-double positive cells were quantified (see Supplemental Figure S2A at http:// ajp.amjpathol.org). A low basal level of phagocytosis was observed in fully myelinated cultures at 21 DIV; numbers of phagocytic cells were significantly increased by 5 DIV postlysolecithin, which subsided significantly by 14 DIV postlysolecithin. Fingolimod treatment (100 pmol/L) significantly decreased the numbers of phagocytosing microglia at both 5 DIV (from $13 \pm 0$ to $5 \pm 2$ ) and 14 DIV postdemyelination (from $4 \pm 1$ to $1 \pm 1$ ) (see Supplemental Figure S2B at http://ajp.amjpathol.org).

\section{Functional Basis for the Effects of Fingolimod During Remyelination}

To investigate the mechanism through which fingolimod enhanced remyelination in demyelinated slice cultures, we compared the effects of co-treating cultures with fingolimod and antagonists of specific S1P receptor subtypes on remyelination efficiency. Co-treatment of cultures with fingolimod (100 pmol/L) and a S1P1 receptor antagonist W123 only slightly decreased remyelination as assessed by MBP immunohistochemistry (from $12.9 \pm$ 
A
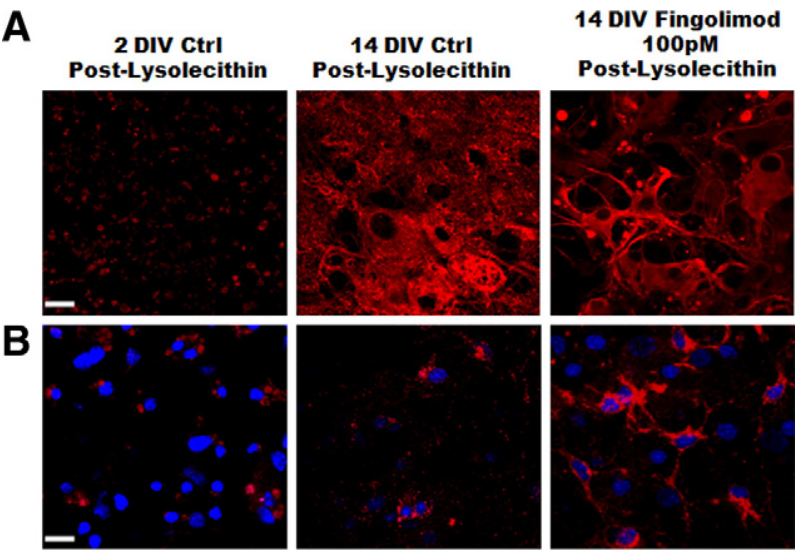

C

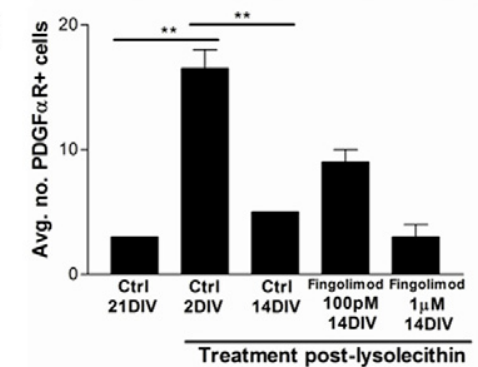

Figure 4. Effects of fingolimod on oligodendroglial lineage cells under remyelinating conditions. A: Representative images of mature oligodendrocytes immunostained against NogoA (red). Cultures at 2 DIV postlysolecithin demonstrate a loss of NogoA immunoreactivity; control cultures at 14 DIV postlysolecithin show some recovery of NogoA immunostaining. Fingolimod treatment for 14 DIV postlysolecithin induces extension of processes in mature oligodendrocytes relative to control. Scale bar $=20 \mu \mathrm{m}$. B: Representative images of OPCs immunostained against PDGF $\alpha$ R (red) and counterstained with the nuclear dye Topro-3 (blue) demonstrate an increase in OPC numbers in cultures 2 DIV postlysolecithin, and a subsequent decrease in control cultures at 14 DIV postlysolecithin likely representing differentiation and loss of the marker. Process extension was observed in OPCs in cultures treated with fingolimod for 14 DIV postlysolecithin. Scale bar $=20$ $\mu \mathrm{m}$. C: Quantification of absolute numbers of PDGF $\alpha \mathrm{R}$ immunopositive OPCs per $\times 63$ objective image \pm SEM. At 2 DIV postlysolecithin, numbers of OPCs are significantly increased compared with pre-lysolecithin (control 21 DIV). These numbers subsequently decrease by 14 DIV postlysolecithin in control cultures. Fingolimod treatment $(100 \mathrm{pmol} / \mathrm{L})$ for 14 DIV postlysolecithin induced a trend toward an increase in OPC numbers. ${ }^{* * *} P<0.01$

4.1 to $7.2 \pm 1.3$ fold over control; Figure $6, \mathrm{~A}$ and $\mathrm{B}$ ). However, fingolimod co-treatment with a S1P3/S1P5 pathway antagonist suramin $(100 \mathrm{nmol} / \mathrm{L})$ significantly reversed the enhanced remyelination observed with fingolimod alone (to $2.5 \pm 1.0$ fold over control; Figure 6 , $A-C)$. These results suggest that fingolimod enhanced remyelination in the cultures primarily through S1P3/S1P5 signaling. Accordingly, remyelination was inhibited and MBP immunoreactivity was associated with myelin debris when a S1P1 agonist SEW2871 (100 nmol/L) was applied alone to the cultures, whereas a trend increase in remyelination was observed with a S1P5 specific agonist (100 $\mathrm{nmol} / \mathrm{L} ; 2.6 \pm 1.6$ fold over control).

We next assessed how these effects of specific S1P receptor blocking and activation correlated to astrocyte and microglial responses in the slice. The increased astrocytic response observed at 14 DIV postlysolecithin with fingolimod treatment was significantly increased when W123 was supplemented to cultures (from 3. $0 \pm$ 0.3 to $7.1 \pm 0.2$ fold over control), whereas suramin supplementation significantly reversed values compared with fingolimod alone (1.3 \pm 0.5 fold over control; Figure 7, A and B). These findings suggest that whereas S1P3/5 signaling promotes astrogliosis, this is inhibited by S1P1 signaling, and blocking the latter further increases the astrocytic response induced by fingolimod. In support of this, SEW2871 applied to demyelinated cultures significantly decreased the area of GFAP immunostaining compared with control $(0.5 \pm 0.1$ fold $)$, whereas a S1P5 agonist induced a trend increase in values $(1.3 \pm 0.5$ fold over control; Figure 7B).

The increase in microglia cell numbers observed at 14 DIV postdemyelination with fingolimod treatment alone (6.4 \pm 0.8 fold over control) were slightly yet not significantly decreased with W123 co-treatment (5.1 \pm 0.5$)$, yet were increased when suramin was supplemented to the cultures $(10.7 \pm 1.0)$. We observed that treatment with SEW2871 or the S1P5 agonist was able to induce a significant increase in microglia cell numbers (6.2 \pm 0.2 and $5.4 \pm 0.4$ fold over control, respectively). Together, the data suggests that signaling through S1P1 and S1P5 can contribute to enhanced microgliosis, yet the increase in cell numbers consequent to S1P3/5 blockade via suramin implies that S1P3 signaling may suppress this response. The increase in microglia cell numbers in the SEW2871-treated condition, where remyelination was inhibited, demonstrates that $\mathrm{S} 1 \mathrm{P}$ receptor-driven enhancement of microgliosis is not sufficient to drive the myelin repair response.

\section{Discussion}

\section{Effect of Fingolimod on Myelin Maintenance}

Long-term treatment (21 DIV) of fully myelinated organotypic cerebellar slice cultures with both low (100 pmol/L) and high ( $1 \mu \mathrm{mol} / \mathrm{L})$ doses of fingolimod did not induce any deleterious effect on myelin maintenance. Fingolimod promoted the elaboration of membranous lamellipodial extensions by OPCs and OLGs, and increased OPC cell number. The elaboration of processes is critical for myelination to proceed ${ }^{45}$ and precise regulation of process extension is required for OPC migration and differentiation into mature OLGs. ${ }^{46-47}$ Our previous studies in vitro demonstrated that fingolimod induces process extension by human-derived OPCs and mature OLGs under basal culture conditions. ${ }^{27-28}$ We demonstrate that fingolimod-induced oligodendroglial membrane elaboration was not sufficient to increase the amount of MBP in the cultures. We hypothesize that all fibers that could be myelinated within the culture may have already been ensheathed thereby preventing any increase in myelination. In contrast with the findings we present here using organotypic slices, rodent OLGs in dispersed cell cultures do not modulate their cytoskeleton in response to the drug, ${ }^{28}$ and fingolimod alone has no effect on rodent and human OPC cell numbers. ${ }^{27,34}$ These findings indicate that OLGs and OPCs may either modulate their S1P receptor levels in an environment containing multiple cell types, or that they are responding to factors being re- 


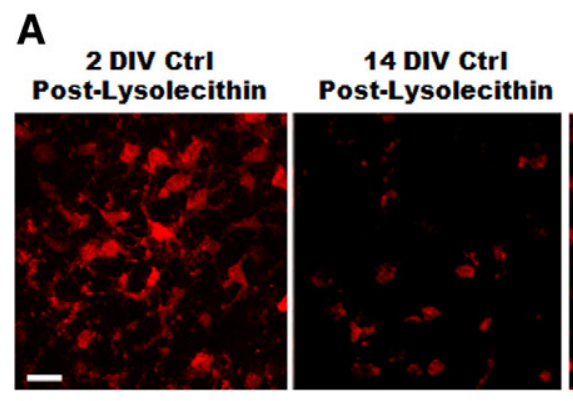

B

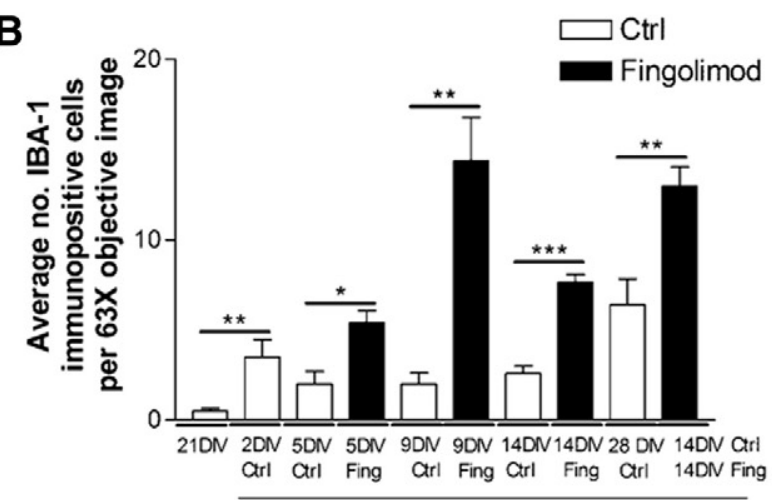

Treatment post-lysolecithin
14 DIV Fingolimod $100 \mathrm{pM}$ Post-Lysolecithin

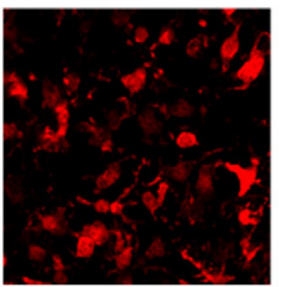

$\square$ Ctrl

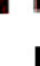

D

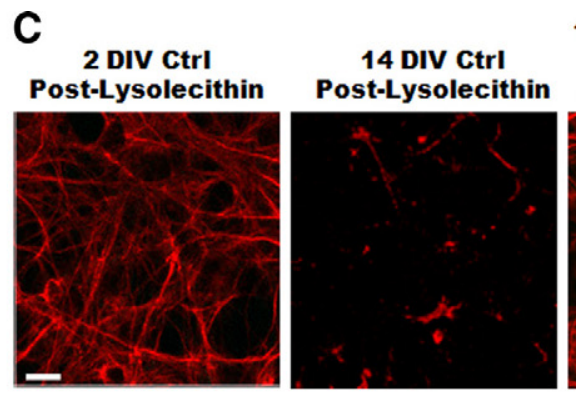

14 DIV Fingolimod 100pM Post-Lysolecithin
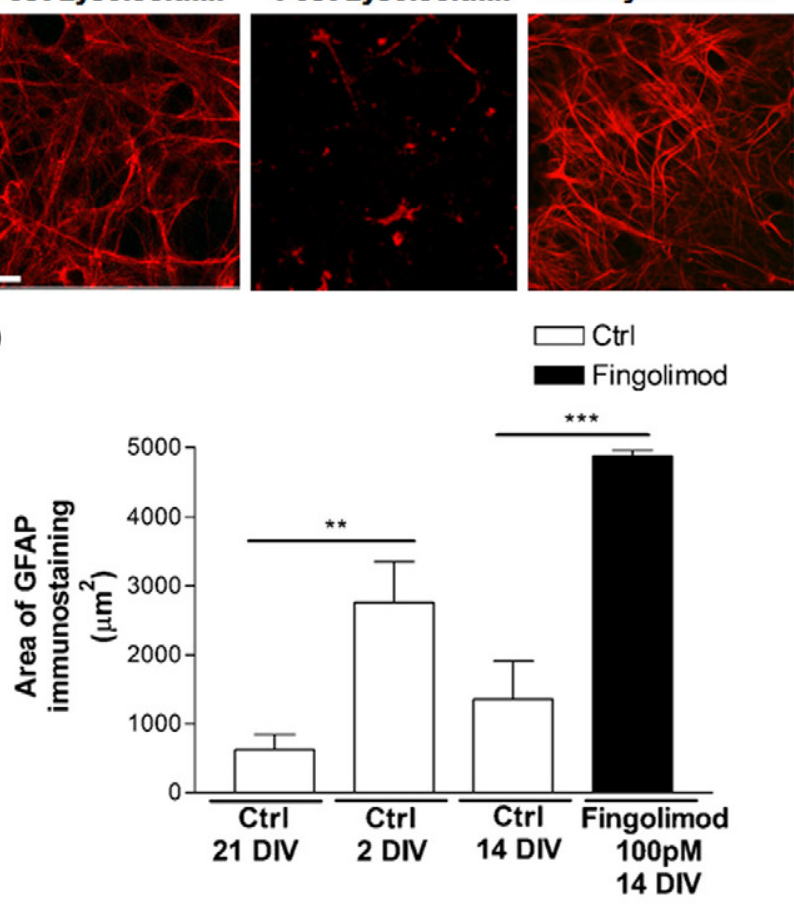

Treatment post-lysolecithin

Figure 5. Effects of fingolimod on microglia and astrocytes under remyelinating conditions. A: Representative images of slices at 35 DIV immunostained against the microglial marker IBA-1 (red). Microglia are increased at 2 DIV postlysolecithin and subsequently decrease by 14 DIV. Cultures treated with fingolimod (100 $\mathrm{pmol} / \mathrm{L}$ ) for $14 \mathrm{DIV}$ postlysolecithin show relatively increased numbers of microglia relative to control cultures. Scale bar $=20 \mu \mathrm{m}$. B: Quantification of numbers of microglia represented as average IBA- 1 immunopositive cells per $\times 63$ objective image \pm SEM. Microglia cell numbers are increased by 2 DIV postlysolecithin, which subsides by 5 DIV. Fingolimod-treated cultures (Fing; black bars) showed a significant increase in microglia cell numbers at 5 , 9, and 14DIV postdemyelination compared with controls (Ctrl; white bars). Delaying 14 DIV fingolimod treatment for 2 weeks following lysolecithin treatment still induced an increase in microglia cell numbers in comparison with control cultures at 28 DIV postlysolecithin. ${ }^{*} P<0.05,{ }^{* *} P<0.01,{ }^{* * * *} P<0.001$. C: Representative images of slices at 35 DIV immunostained against the astrocytic marker GFAP (red). Astrocytes are increased at 2 DIV postlysolecithin and subsequently decrease by 14 DIV. Cultures treated with fingolimod ( $100 \mathrm{pmol} / \mathrm{L})$ for $14 \mathrm{DIV}$ postlysolecithin show relatively increased GFAP immunostaining relative to control cultures. Scale bar $=20 \mu \mathrm{m}$. D: Quantification of astrocytes represented as area of GFAP immunostaining $\left(\mu \mathrm{m}^{2}\right) \pm$ SEM. Demyelination with lysolecithin $(2 \mathrm{DIV}$ postlysolecithin) induces an increase in GFAP staining compared with pre-demyelination controls (21 DIV). In 14 DIV postlysolecithin control cultures, GFAP staining shows a relative decrease compared with 2 DIV postlysolecithin. Fingolimod treatment $(100 \mathrm{pmol} / \mathrm{L})$ for 14 DIV postlysolecithin induces an increase in GFAP staining, as compared with control. ${ }^{* *} P<0.01,{ }^{* * * *} P<0.001$.

leased by other fingolimod-stimulated neuroglial cells in the slice.

\section{Effects of Fingolimod on Remyelination}

Demyelination was induced in cerebellar slice cultures by overnight treatment with lysolecithin, inducing a loss of MBP associated with axons, an increase in MBP immunostaining resembling debris, a decrease in the frequency of nodes of Ranvier, and a lengthening of the nodal region.

The adult CNS undergoes at least partial repair following injury. Myelin repair occurs via remyelination, and is considered to be associated with recovery of axonal function and clinical remittance. ${ }^{13,14,48}$ Studies of organotypic cerebellar slice cultures indicate that following a transient insult with lysolecithin, OPC differentiation and re-expression of myelin proteins occurs in the same temporal succession that would be observed in vivo. ${ }^{37}$ These studies demonstrated that the remyelination process is initiated within 1 week of demyelination, yet remains lim- ited at 11 DIV postdemyelination. ${ }^{37}$ To document remyelination, we used MBP immunohistochemistry, which has previously been used to monitor this process in slice cultures, and co-labels with the late differentiation marker myelin oligodendrocyte glycoprotein by 11 DIV postdemyelination. ${ }^{37}$ Consistent with previous studies, the cerebellar slice cultures used in our current study initiated remyelination within 5 days of demyelination and underwent observable yet incomplete remyelination by 14 DIV postdemyelination. At 14 DIV postdemyelination, treatment with both high and low doses of fingolimod increased the amount of MBP immunostaining in demyelinated cultures in comparison with control. Toluidine-blue stained sections and ultrastructural analysis revealed the presence of many thin myelin sheaths in fingolimodtreated cultures suggestive of remyelination. Our findings using S1P receptor specific agonists and antagonists suggest that fingolimod enhanced remyelination primarily via S1P3/5 signaling; engagement of S1P5 was sufficient to induce a trend increase in remyelination. S1P5 is highly expressed on mature oligodendrocytes, ${ }^{28,29}$ and S1P5- 
A
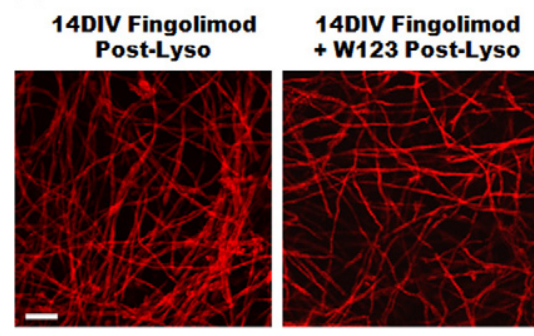

14DIV Fingolimod + Suramin Post-Lyso

B

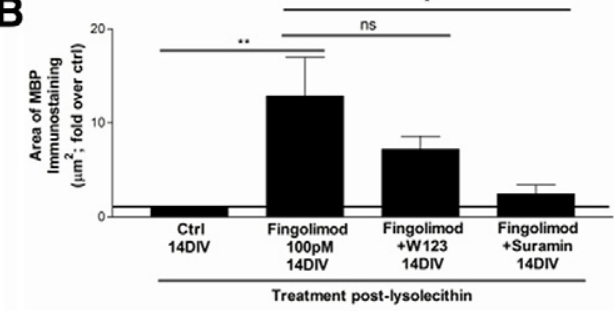

C

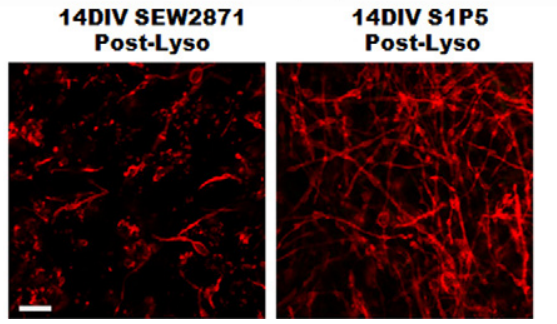

Figure 6. Mechanism of fingolimod-mediated effects on remyelination. A: Representative images of myelin (MBP) in cultures at 14 DIV postdemyelination, treated with fingolimod $(100 \mathrm{pmol} / \mathrm{L})$ alone, or co-treated with a S1P1 receptor antagonist W123 or a $\mathrm{S} 1 \mathrm{P} 3 / 5$ pathway antagonist suramin. Scale bar $=20 \mu \mathrm{m}$. B: Quantification of amount of myelin expressed as area of MBP immunostaining $\left(\mu \mathrm{m}^{2}\right)$ per $\times 63$ objective image normalized to control culture values at 14 DIV postlysolecithin. The increased remyelination observed with fingolimod treatment $(100 \mathrm{pmol} / \mathrm{L})$ for 14 DIV postlysolecithin was slightly decreased with W123 co-treatment, yet was only significantly reversed with suramin supplementation. ${ }^{*} P<0.05$, ${ }^{* *} P<0.01$. C: Representative images of cultures treated with specific S1P receptor agonists for 14 DIV following demyelination. The S1P1 agonist SEW2871 $(100 \mathrm{nmol} / \mathrm{L})$ caused a decrease in the amount of myelin associated with axons and increase of myelin debris. A S1P5 agonist (100 nmol/L) induced trend increases in remyelination. Scale bar $=20 \mu \mathrm{m}$.

mediated signaling has pro-survival effects on human and rodent mature oligodendrocytes. ${ }^{28,29}$ Whereas our studies using dissociated cultures of human OPCs demonstrated that S1P1 signaling could promote process extension, differentiation, and survival, ${ }^{27}$ here we observed that signaling through this receptor inhibited remyelination. One postulate for this unexpected result is that the growth factor environment within the slice may have modulated responses to fingolimod. Previous studies have demonstrated how responses to S1P receptor modulators may be influenced by growth factors, for instance PDGF and S1P receptor signaling are coordinated and this growth factor regulates expression levels of S1P receptors. ${ }^{34}$

The increase in absolute numbers of PDGF $\alpha$ R positive OPCs following lysolecithin treatment is consistent with the previously documented incorporation of BrdU into PDGF $\alpha \mathrm{R}+$ cells in lipopolysaccharide-treated organotypic cultures ${ }^{49}$ and increased OPC proliferation following demyelination in vivo. ${ }^{15}$ Whereas OPC numbers subsequently subsided over time in control cultures, by 14
A
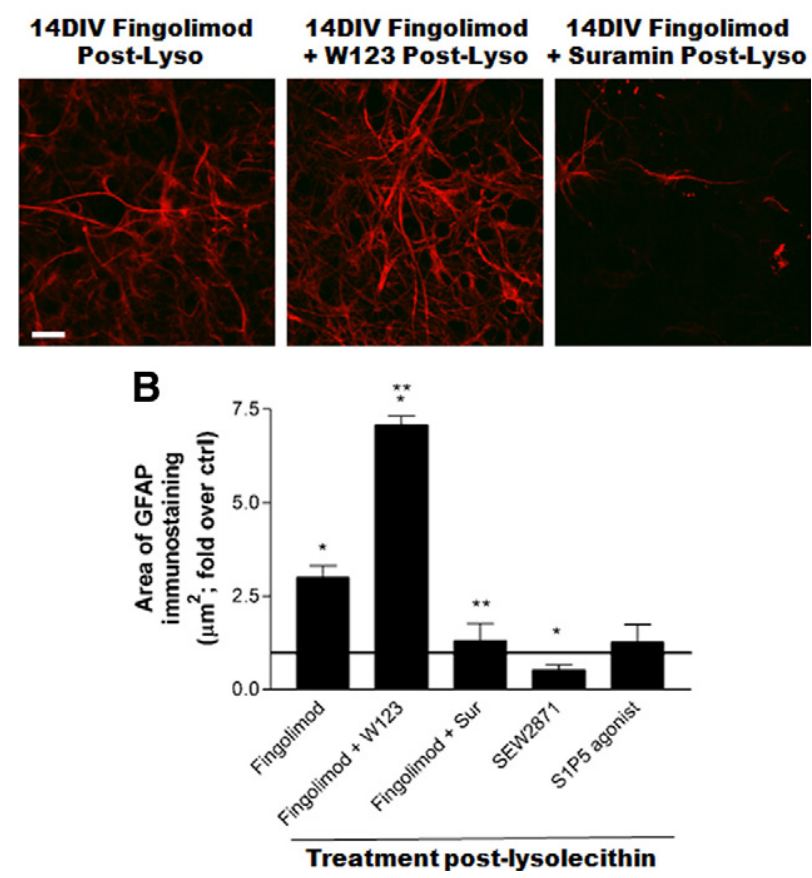

Figure 7. Mechanism of fingolimod-induced effects on astrocytes. A: Representative images of GFAP immunostaining of cultures at 14 DIV postdemyelination. The increase in GFAP immunostaining observed with fingolimod treatment $(100 \mathrm{pmol} / \mathrm{L})$ was further increased when the S1P1 antagonist W123 was supplemented to the cultures, suggesting a disinhibition of S1P3/ 5-associated signaling. Accordingly, suramin supplementation significantly decreased the area of GFAP immunostaining compared with fingolimod alone. Scale bar $=20 \mu \mathrm{m}$. B: Quantification of the astrocytic response represented as area of GFAP immunostaining $\left(\mu \mathrm{m}^{2}\right)$ normalized to control at 14 DIV postdemyelination. Fingolimod-induced increase in values were significantly increased by W123 and reversed with suramin. The S1P1 agonist SEW2871 (100 nmol/L) significantly decreased GFAP immunostaining, as compared with control, whereas a S1P5 agonist (100 nmol/L) had no significant effect. ${ }^{*} P<0.05,{ }^{* * *} P<0.01$.

DIV postlysolecithin, fingolimod treatment induced a trend increase in absolute numbers of OPCs. Increased OPC numbers may have reflected enhanced survival or proliferation, which have been documented to occur in dissociated cultures. ${ }^{27,30,34}$ More OPCs may have been available to participate in remyelination compared with control conditions. We hypothesize that the number of PDGF $\alpha R+$ cells may not have reached significance with fingolimod treatment due to a putative acceleration of OPC differentiation and consequent loss of PDGF $\alpha$ R expression. Fingolimod also induced process extension by both OPCs and OLGs under remyelinating conditions. Low doses of fingolimod induce differentiation of rat OPCs into mature OLGs in dissociated cultures whereas high doses have the opposite effect. ${ }^{30,34}$ In our slices, OPCs may not have been exposed to high enough doses of the drug required to inhibit differentiation, perhaps as a result of sequestration by OLGs/myelin. ${ }^{22}$

\section{Effects of Fingolimod on Microglia and Astrocytes Under Remyelinating Conditions}

We observed that microglial cell numbers peaked at 2 DIV postlysolecithin and declined by 14 DIV postlysole- 
cithin in control cultures. Previous studies have demonstrated the abundance of microglia in these cultures and their activation consequent to demyelination. ${ }^{37}$ Microglia are increased in numbers by 3 days following ethidium bromide-induced demyelination in vivo with concomitant increases in PDGF $\alpha$ R+ OPCs at the lesion border. ${ }^{50}$ Fingolimod-treated cultures demonstrated an increase in microglia cell numbers by 5 DIV following demyelination that was maintained to at least 14 DIV postdemyelination. When the drug application (for 14 DIV) was delayed by 2 weeks, the increase in microglia numbers was still detected, suggesting that its occurrence was not a consequence of a response to demyelination. One key mechanism whereby macrophages/microglia contribute to remyelination involves their capacity to phagocytose myelin debris, as confirmed by depletion of these cells with clodronate-liposomes. ${ }^{16,17}$ Using fluorescently-conjugated bacteria as an index of phagocytic activity, we observed a peak in numbers of phagocytic microglia shortly after demyelination (2 and 5 DIV) in control cultures. In cultures exposed to fingolimod, a decrease in the absolute numbers of actively phagocytic cells was detected at 5 and 14 DIV postlysolecithin. Although fingolimod treatment may have reduced the number of phagocytic microglia, the increase in total numbers of microglia could have been sufficient to result in myelin debris ingestion such that remyelination could occur. Ultrastructural analysis of fingolimod-treated slices indicated the presence of phagocytosed myelin debris; we could not appreciate any qualitative difference in the amount of MBP-immunoreactive debris found in fingolimod-treated cultures compared with control cultures at 14 DIV postdemyelination. Our studies using S1P receptor specific agonists and antagonists suggest that signaling via S1P1 and S1P5 contribute to microgliosis, whereas S1P3mediated signaling may inhibit this response. The increase in microglia cell numbers under treatment conditions that either promoted remyelination (fingolimod, S1P5) or inhibited remyelination (SEW2871) suggest that S1P receptormediated enhancement of microglia numbers may have contributed to, but was not sufficient for, myelin repair.

We observed an increase in GFAP immunoreactivity by 2 DIV following demyelination, which subsided by 14 DIV in control cultures. We observed increased GFAP immunoreactivity at 14 DIV postlysolecithin when fingolimod was applied to cultures. Although astrocytes are associated with formation of a glial scar, destruction of tissue, and production of factors that can inhibit OPC proliferation and maturation (ie, tumor necrosis factor- $\alpha$ ), ${ }^{51}$ they have the capability to produce factors that can enhance OPC function. Astrocytes have been suggested to be beneficial in an acute inflammatory environment soon after demyelination. ${ }^{52}$ Astrocytes stimulated with pro-inflammatory cytokines in vitro or found at the edge of active MS lesions produce growth factors and chemokines that can influence OPC survival, differentiation, migration, and remyelination. ${ }^{18,19,53}$ Furthermore, S1P receptor activation in cultured astrocytes leads to nerve growth factor production. ${ }^{54}$ Injection of exogenous astrocytes into spinal cord lesions enhances remyelination, ${ }^{55}$ and remyelination of such lesions is only seen in the astrocytic perimeter ${ }^{56} \mathrm{~A}$ lack of remyelination has been observed in regions devoid of astrocytes. ${ }^{57}$ Application of S1P receptor specific agonists and antagonists revealed that the fingolimod-induced increase in astrogliosis was mediated primarily through S1P3/S1P5, whereas S1P1 signaling appeared to inhibit this response. In comparison, one study using dissociated astrocyte cultures has implicated S1P1 in astrocyte proliferation. ${ }^{33}$ However, other studies have demonstrated that S1P or fingolimod-mediated signaling and functional effects in dissociated cultures of rodent astrocytes are mediated via $G_{i}{ }^{56,58-60} ; G_{i}$ can be associated with S1P5 activation. ${ }^{61}$ An alternative explanation for this discrepancy is that fingolimod effects on neuroglia within the slice may have caused the release of factors that modulated the astrocytic response. In support of this postulate, S1P receptor engagement on astrocytes can induce growth factor production ${ }^{54,62}$ and growth factors can modulate S1P receptor gene expression in rat astrocytes in an autocrine manner such that S1P5 levels are increased. ${ }^{63}$ This suggests that within a physiological environment, astrocytes may primarily respond to fingolimod via S1P5, rather than through $\mathrm{S} 1 \mathrm{P} 1$ as predicted from isolated cultures. Interestingly, treatment conditions under which astrogliosis was enhanced (fingolimod, S1P5) coincided with those sufficient to ameliorate remyelination, further supporting the beneficial role of astrocytes in the remyelination process.

\section{Conclusion}

Our studies suggest that fingolimod enhanced remyelination, which was well-correlated with an astrocytic response, both mediated via S1P3/S1P5, whereas a S1P1and S1P5-dependent increase in microglia cell numbers and effects on phagocytosis did not appear sufficient to enhance the myelin repair process. These data establish that S1P receptor-specific signaling on multiple neuroglial cell types in a physiological environment can influence remyelination, in a manner that may not have been predicted from dissociated culture studies.

\section{Acknowledgments}

We thank Manon Blain for technical support and Jeannie Mui [Facility for Electron Microscopy Research (FEMR) at McGill University] for processing and cutting tissue for electron microscopy. We also thank Anna Schubart (Novartis Pharma) for helpful discussions.

\section{References}

1. Griffiths I, Klugmann M, Anderson T, Yool D, Thomson C, Schwab $\mathrm{MH}$, Schneider A, Zimmermann F, McCulloch M, Nadon N, Nave KA: Axonal swellings and degeneration in mice lacking the major proteolipid of myelin. Science 1998, 280:1610-1613

2. Lappe-Siefke C, Goebbels S, Gravel M, Nicksch E, Lee J, Braun PE, Griffiths IR, Nave KA: Disruption of Cnp1 uncouples oligodendroglial functions in axonal support and myelination. Nat Genet 2003, 33:366-374

3. Yin X, Baek RC, Kirschner DA, Peterson A, Fujii Y, Nave KA, Macklin 
WB, Trapp BD: Evolution of a neuroprotective function of central nervous system myelin. J Cell Biol 2006, 172:469-478

4. Lajtha A, Toth J, Fujimoto K, Agrawal HC: Turnover of myelin proteins in mouse brain in vivo. Biochem J 1977, 164:323-329

5. LeBaron FN, Sanyal S, Jungalwala FB: Turnover rate of molecular species of sphingomyelin in rat brain. Neurochem Res 1982, 6:1081-1089

6. Trapp BD, Peterson J, Ransohoff RM, Rudick R, Mork S, Bo L: Axonal transection in the lesions of multiple sclerosis. N Engl J Med 1998, 338:278-285

7. Arnold DL: Magnetic resonance spectroscopy: imaging axonal damage in MS. J Neuroimmunol 1999, 98:2-6

8. Bruck W, Kuhlmann T, Stadelmann C: Remyelination in multiple sclerosis. J Neurol Sci 2003, 206:181-185

9. Chen JT, Collins DL, Freedman MS, Atkins HL, Arnold DL: Local magnetization transfer ratio signal inhomogeneity is related to subsequent change in MTR in lesions and normal-appearing white-matter of multiple sclerosis patients. Neuroimage 2005, 25:1272-1278

10. Redford EJ, Smith KJ, Gregson NA, Davies M, Hughes P, Gearing AJ, Miller K, Hughes RA: A combined inhibitor of matrix metalloproteinase activity and tumour necrosis factor-alpha processing attenuates experimental autoimmune neuritis. Brain 1997, 120:1895-1905

11. Kornek B, Lassmann H: Neuropathology of multiple sclerosis-new concepts. Brain Res Bull 2003, 61:321-326

12. Wilkins $A$, Chandran $S$, Compston A: A role for oligodendrocytederived IGF-1 in trophic support of cortical neurons. Glia 2001, 36:48-57

13. Liebetanz D, Merkler D: Effects of commissural de- and remyelination on motor skill behaviour in the cuprizone mouse model of multiple sclerosis. Exp Neurol 2006, 202:217-224

14. Smith PM, Jeffery ND: Histological and ultrastructural analysis of white matter damage after naturally-occurring spinal cord injury. Brain Pathol 2006, 16:99-109

15. Gensert JM, Goldman JE: Endogenous progenitors remyelinate demyelinated axons in the adult CNS. Neuron 1997, 19:197-203

16. Loughlin AJ, Copelman CA, Hall A, Armer T, Young BC, Landon DN, Cuzner ML: Myelination and remyelination of aggregate rat brain cell cultures enriched with macrophages. J Neurosci Res 1997, 47:384392

17. Kotter MR, Setzu A, Sim FJ, Van Rooijen N, Franklin RJ: Macrophage depletion impairs oligodendrocyte remyelination following lysolecithin-induced demyelination. Glia 2001, 35:204-212

18. Dziembowska M, Tham TN, Lau P, Vitry S, Lazarini F, Dubois-Dalq M: A role for CXCR4 signaling in survival and migration of neural and oligodendrocyte precursors. Glia 2005, 50:258-269

19. Zhang Y, Taveggia C, Melendez-Vasquez C, Einheber S, Raine CS, Salzer JL, Brosnan CF, John GR: Interleukin-11 potentiates oligodendrocyte survival and maturation, and myelin formation. J Neurosci 2006, 26:12174-12185

20. Kappos L, Antel J, Comi G, Montalban X, O'Connor P, Polman CH, Haas T, Korn AA, Karlsson G, Radue EW: Oral fingolimod (FTY720) for relapsing multiple sclerosis. N Engl J Med 2006, 355:1124-11240

21. Meno-Tetang GM, Li H, Mis S, Pyszczynski N, Heining P, Lowe P, Jusko WJ: Physiologically based pharmacokinetic modeling of FTY720 (2-amino-2[2-(-4-octylphenyl)ethyl]propane-1,3-diol hydrochloride) in rats after oral and intravenous doses. Drug Metab Dispos 2006, 34:1480-1487

22. Foster CA, Howard LM, Schweitzer A, Persohn E, Hiestand PC, Balatoni B, Reuschel R, Beerli C, Schwartz M, Billich A: Brain penetration of the oral immunomodulatory drug FTY720 and its phosphorylation in the central nervous system during experimental autoimmune encephalomyelitis: consequences for mode of action in multiple sclerosis. J Pharmacol Exp Ther 2007, 323:469-475

23. Billich A, Bornancin F, Devay P, Mechtcheriakova D, Urtz N, Baumruker T: Phosphorylation of the immunomodulatory drug FTY720 by sphingosine kinases. J Biol Chem 2003, 278:47408-47415

24. Zemann B, Kinzel B, Muller M, Reuschel R, Mechtcheriakova D, Urtz $\mathrm{N}$, Bornancin F, Baumruker T, Billich A: Sphingosine kinase type 2 is essential for lymphopenia induced by the immunomodulatory drug FTY720. Blood 2006, 107:1454-1458

25. Yu N, Lariosa-Willingham KD, Lin FF, Webb M, Rao TS: Characterization of lysophosphatidic acid and sphingosine-1-phosphate-mediated signal transduction in rat cortical oligodendrocytes. Glia 2004, $45: 17-27$
26. Novgorodov AS, El Alwani M, Bielawski J, Obeid LM, Gudz TI: Activation of sphingosine-1-phosphate receptor S1P5 inhibits oligodendrocyte progenitor migration. FASEB J 2007, 21:1503-1514

27. Miron VE, Jung CG, Kim HJ, Kennedy TE, Soliven B, Antel JP: FTY720 modulates human oligodendrocyte progenitor process extension and survival. Ann Neurol 2008, 63:61-71

28. Miron VE, Hall JA, Kennedy TE, Soliven B, Antel JP: Cyclical and dose-dependent responses of adult human mature oligodendrocytes to fingolimod. Am J Pathol 2008, 173:1143-1152

29. Jaillard C, Harrison S, Stankoff B, Aigrot MS, Calver AR, Duddy G, Walsh FS, Pangalos MN, Arimura N, Kaibuchi K, Zalc B, Lubetzki C: Edg8/S1P5: an oligodendroglial receptor with dual function on process retraction and cell survival. J Neurosci 2005, 25:1459-1469

30. Coelho RP, Payne SG, Bittman R, Spiegel S, Sato-Bigbee C: The immunomodulator FTY720 has a direct cytoprotective effect in oligodendrocyte progenitors. J Pharmacol Exp Ther 2007, 323:626-635

31. Yamagata K, Tagami M, Torii Y, Takenaga F, Tsumagari S, Itoh S, Yamori Y, Nara Y: Sphingosine 1-phosphate induces the production of glial cell line-derived neurotrophic factor and cellular proliferation in astrocytes. Glia 2003, 41:199-206

32. Pebay A, Toutant M, Premont J, Calvo CF, Venance L, Cordier J, Glowinski J, Tencé M: Sphingosine-1-phosphate induces proliferation of astrocytes: regulation by intracellular signalling cascades. Eur J Neurosci 2001, 13:2067-2076

33. Osinde M, Mullershausen F, Dev KK: Phosphorylated FTY720 stimulates ERK phosphorylation in astrocytes via S1P receptors. Neuropharmacology 2007, 52:1210-1218

34. Jung CG, Kim HJ, Miron VE, Cook S, Kennedy TE, Foster CA, Antel JP, Soliven B: Functional consequences of S1P receptor modulation in rat oligodendroglial lineage cells. Glia 2007, 55:1656-1667

35. Zhang Z, Zhang Z, Fauser U, Artelt M, Burnet M, Schluesener HJ FTY720 attenuates accumulation of EMAP-II+ and MHC-II+ monocytes in early lesions of rat traumatic brain injury. $J$ Cell Mol Med 2007, 11:307-314

36. Jarjour AA, Bull SJ, Almasieh M, Rajasekharan S, Baker KA, Antel JP, Di Polo A, Kennedy TE: Maintenance of axo-oligodendroglial paranodal junctions requires DCC and netrin-1. J Neurosci 2008, 28:11003-11014

37. Birgbauer E, Rao TS, Webb M: Lysolecithin induces demyelination in vitro in a cerebellar slice culture system. J Neurosci Res 2003, 78:157-166

38. Haydar TF, Bambrick LL, Krueger BK, Rakic P: Organotypic slice cultures for analysis of proliferation, cell death, and migration in the embryonic neocortex. Brain Res Brain Res Protoc 1999, 4:425-437

39. Cho S, Wood A, Bowlby MR: Brain slices as models for neurodegenerative disease and screening platforms to identify novel therapeutics. Curr Neuropharmacol 2008, 5:19-33

40. Kuhlmann T, Remington L, Maruschak B, Owens T, Bruck W: Nogo-A is a reliable oligodendroglial marker in adult human and mouse CNS and in demyelinated lesions. J Neuropathol Exp Neurol 2007, 66:238-246

41. Kuhlmann T, Miron V, Cui Q, Wegner C, Antel J, Bruck W: Differentiation block of oligodendroglial progenitor cells as a cause for remyelination failure in chronic multiple sclerosis. Brain 2008, 131:17491758

42. Hall SM: The effect of injections of lysophosphatidyl choline into white matter of the adult mouse spinal cord. J Cell Sci 1972, 10:535-546

43. Blakemore WF, Eames RA, Smith KJ, McDonald WI: Remyelination in the spinal cord of the cat following intraspinal injections of lysolecithin. J Neurol Sci 1977, 33:31-43

44. Coman I, Aigrot MS, Seilhean D, Reynolds R, Girault JA, Zalc B, Lubetzki C: Nodal, paranodal and juxtaparanodal axonal proteins during demyelination and remyelination in multiple sclerosis. Brain 2006, 129:3186-3195

45. Sloane JA, Vartanian TK: WAVE1 and regulation of actin nucleation in myelination. Neuroscientist 2007, 13:486-491

46. Kirby BB, Takada N, Latimer AJ, Shin J, Carney TJ, Kelsh RN, Appel $B$ : In vivo time-lapse imaging shows dynamic oligodendrocyte progenitor behavior during zebrafish development. Nat Neurosci 2006, 9:1506-1511

47. Simons M, Trotter J: Wrapping it up: the cell biology of myelination. Curr Opin Neurobiol 2007, 17:533-540

48. Chen JT, Collins DL, Atkins HL, Freedman MS, Arnold DL: Magneti- 
zation transfer ratio evolution with demyelination and remyelination in multiple sclerosis lesions. Ann Neurol 2008, 63:254-262

49. Filipovic R, Zecevic N: Interaction between microglia and oligodendrocyte cell progenitors involves golli proteins. Ann NY Acad Sci 2005, 1048:166-174

50. Li WW, Setzu A, Zhao C, Franklin RJ: Minocycline-mediated inhibition of microglia activation impairs oligodendrocyte progenitor cell responses and remyelination in a non-immune model of demyelination. J Neuroimmunol 2005, 158:58-66

51. Cammer W, Zhang H: Maturation of oligodendrocytes is more sensitive to TNF alpha than is survival of precursors and immature oligodendrocytes. J Neuroimmunol 1999, 97:37-42

52. Williams A, Platon G, Lubetzki C: Astrocytes-friends or foes in multiple sclerosis? Glia 2007, 55:1300-1312

53. Stankoff B, Aigrot MS, Noel F, Wattiliiaux A, Zalc B, Lubetzki C: Ciliary neurotrophic factor (CNTF) enhances myelin formation: a novel role for CNTF and CNTF-related molecules. J Neurosci 2002, 22:92219227

54. Furukawa A, Kita K, Toyomoto M, Fujii S, Inoue S, Hayashi K, Ikeda K: Production of nerve growth factor enhanced in cultured mouse astrocytes by glycerophospholipids, sphingolipids, and their related compounds. Mol Cell Biol 2007, 305:27-34

55. Blakemore WF, Gilson JM, Crang AJ: The presence of astrocytes in areas of demyelination influences remyelination following transplantation of oligodendrocyte progenitors. Exp Neurol 2003, 184:955-963

56. Bassi R, Anelli V, Giussani P, Tettamanti G, Viani P, Riboni L: Sphingosine-1-phosphate is released by cerebellar astrocytes in response to bFGF and induces astrocyte proliferation through Gi-protein-coupled receptors. Glia 2006, 53:621-630
57. Talbott JF, Loy DN, Liu Y, Qiu MS, Bunge MB, Rao MS, Whittemore SR: Endogenous Nkx2.2+/Olig2+ oligodendrocyte precursor cells fail to remyelinate the demyelinated adult rat spinal cord in the absence of astrocytes. Exp Neurol 2005, 192:11-24

58. Rouach N, Pébay A, Même W, Cordier J, Ezan P, Etienne E, Giaume C, Tencé M: S1P inhibits gap junctions in astrocytes: involvement of G and Rho GTPase/ROCK. Eur J Neurosci 2006, 23:1453-1464

59. Mullershausen F, Craveiro LM, Shin Y, Cortes-Cros M, Bassilana F, Osinde M, Wishart WL, Guerini D, Thallmair M, Schwab ME, Sivasankaran R, Seuwen K, Dev KK: Phosphorylated FTY720 promotes astrocyte migration through sphingosine-1-phosphate receptors. J Neurochem 2007, 102:1151-1161

60. Giussani P, Ferraretto A, Gravaghi C, Bassi R, Tettamanti G, Riboni L, Viani P: Sphingosine-1-phosphate and calcium signaling in cerebellar astrocytes and differentiated granule cells. Neurochem Res 2008, 32:27-37

61. Malek RL, Toman RE, Edsall LC, Wong S, Chiu J, Letterle CA, Van Brocklyn JR, Milstien S, Spiegel S, Lee NH: Nrg-1 belongs to the endothelial differentiation gene family of $G$ protein-coupled sphingosine-1-phosphate receptors. J Biol Chem 2001, 276:5692-5699

62. Sato K, Ishikawa K, Ui M, Okajima F: Sphingosine 1-phosphate induces expression of early growth response- 1 and fibroblast growth factor-2 through mechanism involving extracellular signal-regulated kinase in astroglial cells. Brain Res Mol Brain 1999, 74:182-189

63. Rao TS, Lariosa-Willingham KD, Lin FF, Yu N, Tham CS, Chun J, Webb M: Growth factor pre-treatment differentially regulates phosphoinositide turnover downstream of lysophospholipid receptor and metabotropic glutamate receptors in cultured rat cerebrocortical astrocytes. Int J Dev Neurosci 2004, 22:131-135 